\title{
Composition dependent non-ideality in aqueous binary mixtures as a signature of avoided spinodal decomposition
}

\author{
SARMISTHA SARKAR ${ }^{\mathrm{a}, \mathrm{b}}$, SAIKAT BANERJEE ${ }^{\mathrm{a}}$, SUSMITA ROY ${ }^{\mathrm{a}}$, RIKHIA GHOSH ${ }^{\mathrm{a}}$, \\ PARTHA PRATIM RAY ${ }^{\mathrm{b}}$ and BIMAN BAGCHI ${ }^{\mathrm{a}, *}$ \\ ${ }^{a}$ Solid State and Structural Chemistry Unit, Indian Institute of Science, Bangalore 560 012, India \\ ${ }^{b}$ Department of Physics, Jadavpur University, Kolkata 700 032, India \\ e-mail: profbiman@gmail.com; bbagchi@sscu.iisc.ernet.in
}

MS received 10 June 2014; revised 6 August 2014; accepted 11 August 2014

\begin{abstract}
We explore the potential energy landscape of structure breaking binary mixtures (SBBM) where two constituents dislike each other, yet remain macroscopically homogeneous at intermediate to high temperatures. Interestingly, we find that the origin of strong composition dependent non-ideal behaviour lies in its phase separated inherent structure. The inherent structure (IS) of SBBM exhibits bi-continuous phase as is usually formed during spinodal decomposition. We draw analogy of this correlation between non-ideality and phase separation in IS to explain observation of non-ideality in real aqueous mixtures of small amphiphilic solutes, containing both hydrophilic and hydrophobic groups. Although we have not been able to obtain IS of these liquids, we find that even at room temperature these liquids sustain formation of fluctuating, transient bicontinuous phase, with limited lifetime $(\tau \lesssim 20 \mathrm{ps})$. While in the model (A, B) binary mixture, the non-ideal composition dependence can be considered as a fluctuation from a phase separated state, a similar scenario is expected to be responsible for the unusually strong non-ideality in these aqueous binary mixtures.
\end{abstract}

Keywords. Non-ideality; binary mixture; inherent structures; spinodal decomposition; computer simulation.

\section{Introduction}

The study of aqueous amphiphilic solutions creates an important arena in the research of biology and chemistry. ${ }^{1,2}$ The amphiphilic solutes possess a combination of both hydrophobic and hydrophilic groups allowing them to interact with biopolymers and large molecules in different ways. Examples of some amphiphilic aqueous solvents include water and dimethyl sulfoxide (DMSO), water-methanol, waterethanol, water-tertiary butyl alcohol (TBA), waterdioxane, and water-glycerol, to name a few. Thus combined with water, these amphiphilic co-solvents show some exceptional properties that are of great importance in various fields. ${ }^{3-8}$ The composition dependent non-ideality has been reflected in a number of physical properties such as viscosity, dielectric constant, density, translational and rotational diffusion constants, excess mixing volume, surface tension, heat of formation, etc. ${ }^{9-11}$ However the anomalous behaviours of such aqueous amphiphilic solutions still require further understanding in the molecular level.

The origin of the anomalous behaviours in the real world of aqueous amphiphilic solute molecules can

${ }^{*}$ For correspondence be explained in terms of the structure-making and structure-breaking abilities of the solute-solvent binary mixture. ${ }^{12,13}$ In order to understand many of their unusual physical properties two types of models have been introduced. Model I encourages structure formation whereas Model II encourages structure-breaking, upon mixing. The binary liquids in which molecules of the solute and solvent attract each other are known as structure making/forming binary mixtures and those in which they repel each other are known as structure breaking binary mixtures. The concept of structure formation and breaking in the context of ion mobility can be defined in a different way. Small ions (like $\mathrm{Na}^{+}$) can act as structure maker as they do not disrupt the H-bond network of water molecules and they create certain pockets of solvation spheres where the mobility of water molecules is slow. On the other hand, when the sizes of ions (like $\mathrm{Cs}^{+}$) are larger than that of water molecules, they can interfere with the extended H-bond network and act as structure breaker. ${ }^{2}$ Most of the earlier studies were restricted to structure-forming binary mixture.

Energy landscape view of inherent structure (IS) of structure-breaking liquid is quite different from structure-making liquid ${ }^{14-17}$ IS of the structure breaker is found to be always phase separated where as IS 
of structure-making liquid is homogeneous. This feature of phase separation is similar to the case of liquid crystal where the IS of an isotropic parent phase is found to be nematic ${ }^{18}$ all the time. In fact, inherent structure has been used as an insightful parameter to study the structure and slow dynamics of liquid crystals, ${ }^{18}$ super-cooled liquids and glasses ${ }^{19-21}$ since the innovative work of Stillinger and Weber. ${ }^{22-24}$ This approach to problem of disordered systems has come to be known as the energy landscape view. The configurations of the system corresponding to the local potential energy minima are called the inherent structure (IS). IS can be obtained by removing the kinetic energy, including vibrations, of the atoms and molecules comprising the system and is identified in the simulation by a steepest descent minimization of the potential energy. Many remarkable theoretical studies are based on this energy landscape view. ${ }^{25-28}$ A deeper understanding of much discussed slow dynamics in spinodal decomposition ${ }^{29-31}$ can be obtained through inherent structure analysis.

The kinetics of phase separation proceeds through two mechanisms ${ }^{32}$ in structure breaking binary mixture. In the metastable region of the phase diagram, the phase separation occurs through nucleation while in the unstable region it proceeds through spinodal decomposition. The extensive area of nucleation and spinodal decomposition in binary mixtures has been a subject of great interest in recent times. ${ }^{33-37}$ It is seen that, at least for gas-liquid nucleation, the classical nucleation theory does not work because the composition of the critical nucleus is often quite different from that of the homogeneous mixture. This implies that due to the interplay between surface tension and bulk free energy, nucleation can proceed through intermediate composition states. It is difficult to understand these intermediate states. Surely they constitute fluctuation of the homogeneous mixture, but due to the complexity of binary mixtures, their occurrence is hard to describe.

Recently, the role of spinodal decomposition in crystallization has been explored within a theory. ${ }^{38}$ Phase separation by spinodal decomposition has been described by Cahn-Hillard-Langer-Baron-Miller (CHLBM) theories. ${ }^{39,40}$ In accordance with CHLBM theories, the stability of the system to infinitesimal composition fluctuation can be quantified by the position and time-dependent order parameter $C(\mathbf{r}, t)$ as

$$
C(\mathbf{r}, t)=x_{A}(\mathbf{r}, t)-x_{B}(\mathbf{r}, t),
$$

where, $x_{A}(\mathbf{r}, t)$ and $x_{B}(\mathbf{r}, t)$ are the local, position (r) dependent mole fractions for the solvent and solute, respectively. In a recent study ${ }^{41}$ we have shown the variation of the order parameter $[\mathrm{C}(\mathrm{r})]$ with position. The difference in mole fractions between solvent and solute $[\mathrm{C}(\mathrm{r})]$ with respect to position has been shown to be oscillatory in nature to quantify the signature of spinodal decomposition in the same study. The compositional fluctuations may be considered as the key factors in the initial kinetics of phase separation.

However, a microscopic or even a mesoscopic understanding of the origin of the fluctuations involved in the processes remains somewhat obscure. Recently a systematic investigation leading to a quantitative understanding of the composition dependent non-ideality in binary mixtures has been initiated by using mode coupling theory. ${ }^{12}$ It has been shown that by means of simple model systems where two components interact with disparate interaction potential, one can indeed reproduce much of the non-ideality observed in experiments. One also reproduces many of the features by using mode coupling theory where intermolecular correlations may be taken from simulations. This statistical mechanical approach however cannot explain satisfactorily the correlation between composition fluctuation and phase separation kinetics or even the nature of phase separation in binary mixtures.

In order to get a suitable explanation for the correlation between the phase separation kinetics and composition fluctuation, it has been shown in an earlier study ${ }^{42}$ that the inherent structure of the structure breaker is found to be phase separated in many cases whereas the parent phase is homogeneous. In the same study, when the configurational entropy of the parent liquid has been computed for the two model systems, it has been found that the configurational entropy also shows an inverse correlation with the viscosity in both the cases.

In the recent study, we have presented more detailed analysis of inherent structures (IS) of binary mixture in larger systems and also studied the energy distribution of the IS. ${ }^{41}$ We have reported several highly interesting results. We have found a correlation between the energy of the IS of the binary mixture and the microscopic mode of phase separation in the IS. Structure breakers are shown to exhibit a wide range of structures ranging from nucleation to spinodal decomposition in the IS.

In this work, we demonstrate energy landscape view by simulation of structure breaking binary mixtures. The inherent structure shows spinodal decomposition like pattern formation. We show that the single particle potential energy distribution for parent liquid and inherent structure of structure breaker model suggests partial fleeting phase separation. Moreover, we present the results of many aqueous binary mixtures where the cosolvent is an amphiphilic solute exhibiting pronounced composition dependent anomalies and interestingly a percolation transition leads to the formation of a 
transient bi-continuous phase. We propose that anomalies are due to such avoided spinodal decomposition. The present study establishes a firm relationship between non-ideality and phase separation in binary mixtures.

The organization of the rest of the paper is as follows. In section 2 we describe details of the simulation along with the model systems. In section 3, we present several results on model systems including non-ideality in terms of real binary mixtures where the co-solvent is an amphiphilic solute. In Section 4, we furnish summary of the importance of the results along with concluding remarks.

\section{Computational-Simulation details}

\subsection{Details of the models: Structure former and structure breaker}

We have considered two extreme types of binary mixture models, model I (Structure former) and model II (Structure breaker) to investigate the origin of nonideality. The advantage of our present model is that it can work as the initial point to understand many anomalous properties of binary mixtures. Extensive micro canonical (NVE) ensemble molecular dynamics (MD) simulations have been carried out. Our model binary system consists of total 500 particles [solvent A + solute B] enclosed in a cubic box and periodic boundary conditions are applied. In present models, all the three interactions such as solute-solute $\left(\varepsilon_{\mathrm{BB}}\right)$, solventsolvent $\left(\varepsilon_{\mathrm{AA}}\right)$ and solute-solvent $\left(\varepsilon_{\mathrm{AB}}\right)$ are described by the Lennard-Jones (12-6) potential,

$$
U_{i j}=4 \varepsilon_{i j}\left[\left(\frac{\sigma_{i j}}{r_{i j}}\right)^{12}-\left(\frac{\sigma_{i j}}{r_{i j}}\right)^{6}\right] .
$$

Here ' $\mathrm{i}$ ' and ' $\mathrm{j}$ ' denote any two particles. The diameter $(\sigma)$ and mass $(\mathrm{m})$ for both solute and solvent have been set to unity. The interaction strengths $\varepsilon_{\mathrm{AA}}=1.0$ and $\varepsilon_{\mathrm{BB}}=0.5$ are same for both models, whereas $\varepsilon_{\mathrm{AB}}=$ 2.0 for model I and $\varepsilon_{\mathrm{AB}}=0.3$ for model II (table 1 ). Reduced temperature $\mathrm{T}^{*}=\mathrm{k}_{\mathrm{B}} \mathrm{T} / \varepsilon_{\mathrm{AA}}$ is 1.0 for model I and 1.6 for model II. The reason behind consideration

Table 1. Interaction strengths between solvent $A$ and solute B for structure former and structure breaker model are given below.

\begin{tabular}{lcc}
\hline & Model I & Model II \\
\hline$\varepsilon_{\mathrm{AA}}$ & 1.0 & 1.0 \\
$\varepsilon_{\mathrm{AB}}$ & 2.0 & 0.3 \\
$\varepsilon_{\mathrm{BB}}$ & 0.5 & 0.5 \\
\hline
\end{tabular}

of high temperature for model II is that any microscopic calculation of inherent structure of a binary mixture has to deal with broad phase separation region where solvent (A) and solute (B) components do not like each other for model II. In our structure breaker model we consider the interactions among all particles. However, we also study inherent structure analysis for a comparatively bigger system with 2048 particles, employing nearest neighbour list. The results do not show any significant difference in phase separation patterns.

We have selected an integration time step of $\Delta \mathrm{t}=$ $0.001 \tau$ where the reduced time $\tau$ is defined as $\tau=$ $\sigma \sqrt{\frac{m}{\epsilon_{A A}}}$. We have dealt with different solute compositions from 0.0 to 1.0 in steps of 0.1 . For each solute composition the system has been equilibrated in canonical ensemble for 500000 steps. Next we have carried out simulations for another 2 million steps in microcanonical ensemble and calculate all the relevant properties from this trajectory. Equilibration means the preliminary molecular dynamics simulation to equilibrate the system at the desired temperature. In order to reach equilibration, we scale velocities to reach the chosen temperature. To test whether the system is equilibrated we plot thermodynamic quantity such as temperature as a function of time. If equilibrated, temperature fluctuates around average values. To ensure full equilibration, the MD run has been done initially at higher temperature than the desired value. Non-ideality has always been studied as a function of A and B compositions in the literature of binary mixture. As usual, we have studied the system as a function of the A and B compositions.

\subsection{Computing inherent structures}

In this work, emphasis has been given on the energies and structures of the inherent states which are the local minima of the system obtained by removing all the kinetic energy of the atoms and molecules constituting the system. We have determined inherent structures by quenching the equilibrium configurations to their local minima using conjugate gradient (CG) algorithm. ${ }^{43} \mathrm{CG}$ minimizations were carried out for 2000 equilibrium configurations from which average inherent structure energies have been computed.

\section{Results and Discussion}

\subsection{Non-linear composition dependence of inherent structure energy}

Figures 1 and 2 show the variations of the average inherent structure energy with different solute compositions 

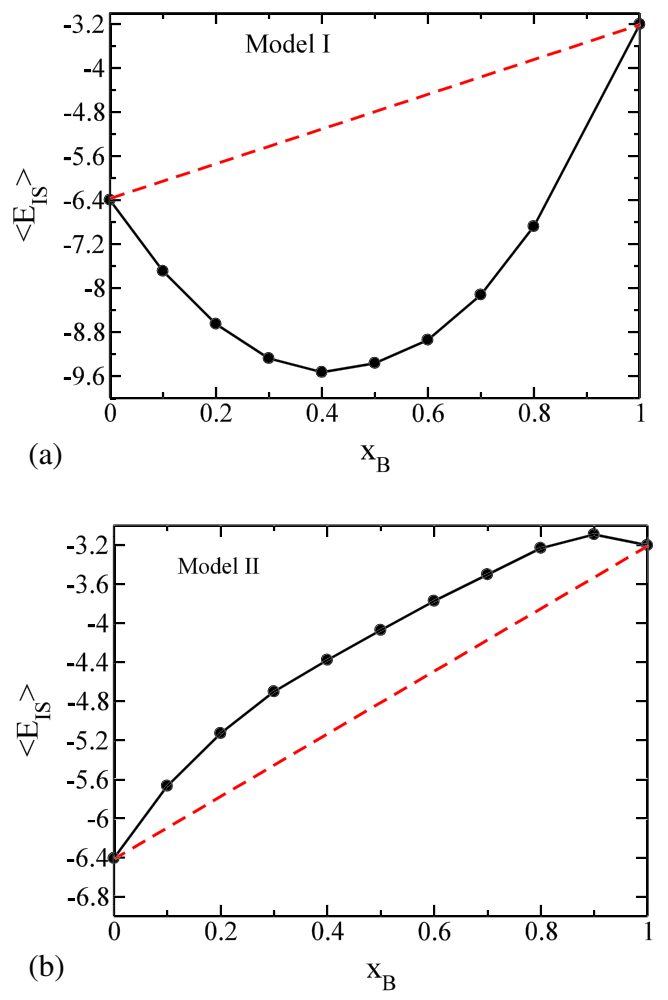

Figure 1. (a) Plot of the computed average inherent structure energy at different solute mole fractions for model I (structure former). The average inherent structure energy $\left(<\mathrm{E}_{\mathrm{IS}}>\right)$ ) shows a minimum at the solute composition $\mathrm{x}_{\mathrm{B}}=0.4$. (b) Plot of $<\mathrm{E}_{\mathrm{IS}}>$ at different solute mole fractions for the model II (structure breaker). In the figure, red line (dark grey) represents the ideal Raoult's law.

for models I and II, respectively. For model I, the average inherent structure energy $\left(<\mathrm{E}_{\mathrm{IS}}>\right)$ shows a minimum at solute composition $\mathrm{x}_{\mathrm{B}}=0.4$. In our previous study, ${ }^{41}$ we presented the calculation of viscosity with respect to mole fraction. The value of viscosity shows a maximum at the solute composition $\mathrm{x}_{\mathrm{B}}=0.4$. This signifies that there is a slowing down of dynamics at

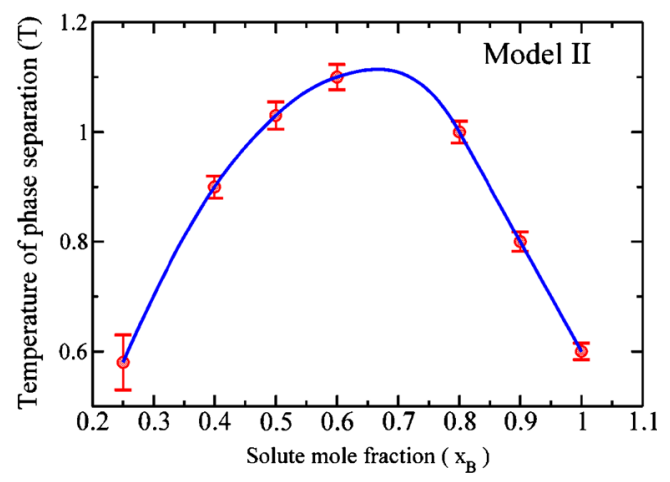

Figure 2. Computed phase diagram of the structure breaker model system. It is evident that the present phase diagram manifests itself as the familiar inverted parabolic shape in the solute composition-temperature plane. Error bars are given. this particular composition $\left(\mathrm{x}_{\mathrm{B}}=0.4\right)$ as the system is ordered here and the system is more structured at the molecular level.

Model II shows exactly the opposite trend. For model II, the average inherent structure energy vs. solute composition curve shows a maximum and the energy lies entirely above the linear line which would signify Raoult's law behaviour. The relatively smaller deviation from ideal behaviour for model II suggests that potential energy landscape is comparatively flat.

\subsection{Phase diagram of the structure breaker model}

Note that one can use the familiar method of free energy calculation (like umbrella sampling) to obtain a phase diagram. Such an approach was used earlier in our study of polydisperse liquids. ${ }^{44}$ In this work, however, we employ a simple approach. Here we have carried out inherent structure analysis to obtain the phase diagram. We have also shown in our previous study that method of IS energy analysis can provide accurate phase diagram. ${ }^{44}$ Additionally, we have taken the help of visual molecular dynamics (VMD) ${ }^{45}$ to get many snapshots for a particular mole fraction and particular temperature to obtain the present phase diagram. VMD is a molecular visualization program for displaying, animating, and analyzing a system from different angular view using 3-D graphics. After analyzing a number of snapshots at closely spaced temperature and mole fraction visually from different angular view, we have achieved the phase diagram. The phase diagrams, obtained from two above mentioned methods are in good agreement with each other.

The same technique has been taken for different solute mole fractions to achieve the simulated phase diagram of the structure breaker binary mixture, as shown in figure 2 . The diagram depicts the familiar inverted parabolic shape in the temperature-composition plane. Although the present phase diagram reflects the well-known inverted parabolic shape, the predicted temperatures of phase separation may not reproduce the precise values in the solute composition-temperature $\left(\mathrm{x}_{\mathrm{B}}, \mathrm{T}\right)$ plane. Also note that the error bars shown in the phase diagram reflect the above uncertainty in our estimate.

\subsection{Distribution of inherent structure energy: Model I and model II}

Figure 3a shows the distribution of inherent structure energies for model I at solute mole fraction $\mathrm{x}_{\mathrm{B}}=0.2$ and $\mathrm{T}^{*}=1.0$ while figure $3 \mathrm{~b}$ shows the distribution of 


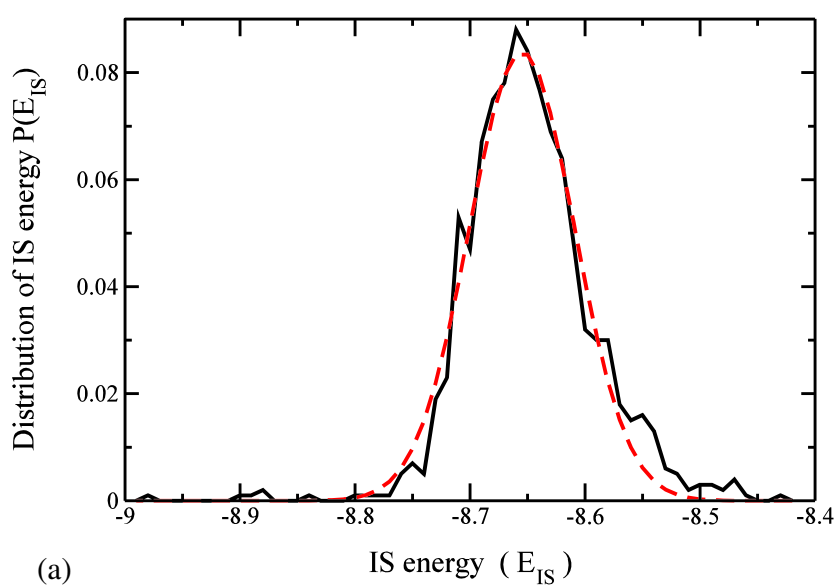

(a)

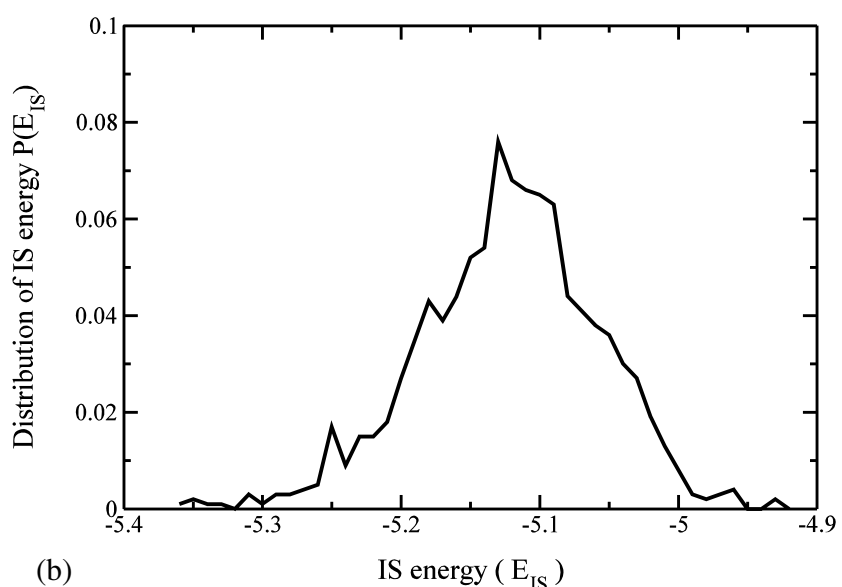

Figure 3. (a) Distribution of inherent structure energy fitted with Gaussian at solute mole fraction $\mathrm{x}_{\mathrm{B}}=0.2$ and $\mathrm{T}^{*}=1.0$ for structure former (model I) binary mixture. Here dotted red colour line represents Gaussian distribution and dashed black line for the distribution of inherent structure. (b) Distribution of inherent structure energy at mole fraction $\mathrm{x}_{\mathrm{B}}=0.2$ and temperature $\mathrm{T}^{*}=1.6$ for structure breaker (model II) binary mixture. Note that this distribution could not be fitted to a Gaussian form.

inherent structure energies for model II at solute mole fraction $\mathrm{x}_{\mathrm{B}}=0.2$ and $\mathrm{T}^{*}=1.6$. The distribution of inherent structure energy for model II is found to be wider compared to that of model I. The curve of IS energies of model I can be fitted to a Gaussian distribution, but the same cannot be done for Model II. Averaging over larger number (2000) of configurations, the IS energy distribution remained noisy for structure breaker model. The values of the standard deviation of the distribution are 0.1396 and 0.7602 for model I and model II, respectively. The wide distribution of structure breaker model suggests strong heterogeneity which is absent in case of model I. The broader inherent structure energy distribution for model II indicates larger entropic contribution towards the stability and homogeneity of structure breaker model. The distribution of average energy of the inherent structure of model I is significantly narrower than that of model II, indicating a larger enthalpic stabilization for structure promoter model. Thus, the homogeneous state of structure breaking solute/solvent system is stabilized by entropy while that of structure former is stabilized by enthalpy.

\subsection{Single particle energy distributions in inherent structure of structure breaker model}

$\mathrm{A}$ and $\mathrm{B}$ components in the parent liquid of structure former are homogeneously distributed across the binary mixture. Even, the IS of the structure former does not show any remarkable heterogeneity. Hence, in order to gain further insight into the microscopic origin of phase separation behaviour, we analyze the structure breaker model from the angle of the single particle distribution of inherent structure energy instead of taking the average of IS energy. We focus each particle separately to look into its individual environment and calculate single particle distribution of parent potential energy and that of corresponding IS energy. Figure 4a shows single particle potential energy distribution of parent liquid for model II at mole fraction $\left(\mathrm{x}_{\mathrm{B}}\right)=0.4$ and temperature $\left(\mathrm{T}^{*}\right)=1.6$ where as figure $4 \mathrm{~b}$ represents the corresponding single particle inherent structure energy distribution. These distributions clearly disclose the existence of dynamic heterogeneity.

\subsection{The molecular arrangements of inherent structure with transient bi-continuous phase}

In order to investigate the origin of diverse non-ideal behaviour of binary mixture, we look into the molecular arrangements of parent structure and the corresponding IS of SBBM. We have taken several snapshots of the parent liquid and the corresponding IS at reduced temperature $\mathrm{T}^{*}=1.6$ with mole fraction $\mathrm{x}_{\mathrm{B}}=0.4$ (figure 5). An instantaneous parent structure is shown in figure $5 \mathrm{a}$ while figure $5 \mathrm{~b}$ and $\mathrm{c}$ depict molecular arrangements for the corresponding inherent structure shown from different angles. While figure 5a does not show any large scale phase separation, figures $5 \mathrm{~b}$ and c indicate a clear signal of spinodal decomposition showing beautiful inter-penetrating phase separation patterns of the constituent atoms A and B.

Although the parent liquid seems to be almost homogeneous over a long time and length scale, there is a clear signature of phase separation in the potential energy minima of the parent structure. Interestingly, the IS of the parent binary liquid shows fleeting bi-continuous pattern formation. The presence of the interconnected percolating networks act as the driving 


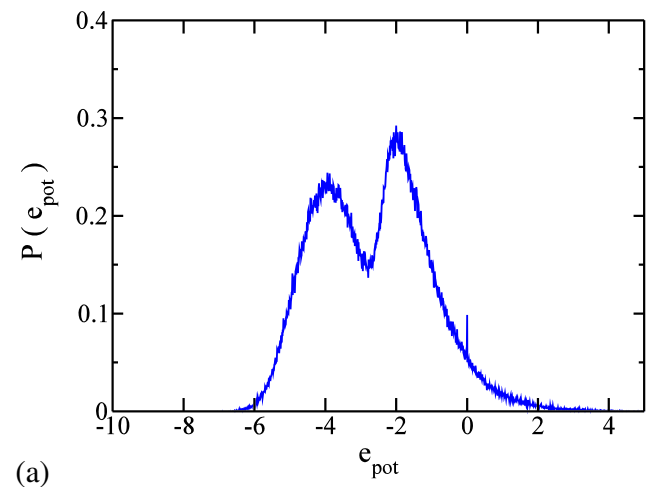

(a)

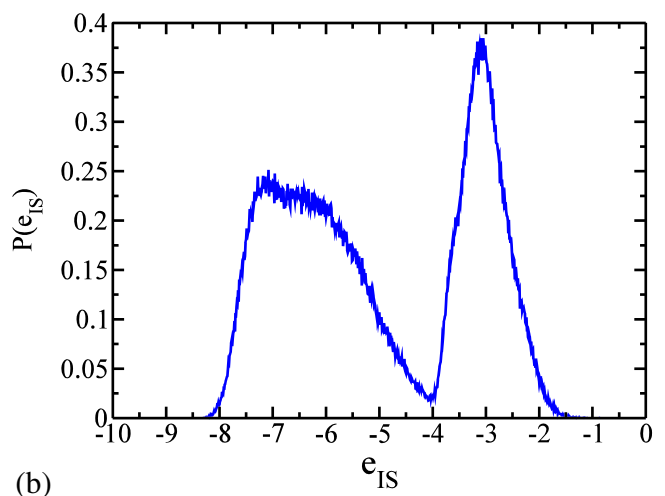

Figure 4. (a) Single particle potential energy distribution, $\mathrm{P}\left(\mathrm{e}_{\mathrm{pot}}\right)$ for structure breaker model at mole fraction $\left(\mathrm{x}_{\mathrm{B}}\right)=$ 0.4 and temperature $\left(\mathrm{T}^{*}\right)=1.6$. It is interesting to note that the parent structure of SBBM is heterogeneous in nature. (b) Single particle inherent structure energy distribution, $\mathrm{P}\left(\mathrm{e}_{\mathrm{IS}}\right)$ for structure breaker model at mole fraction $\left(\mathrm{x}_{\mathrm{B}}\right)=0.4$ and temperature $\left(\mathrm{T}^{*}\right)=1.6$. Note that the single particle inherent structure energy distribution shows that there is a lot of heterogeneity in the IS. Prominent peaks, as shown in figure, reveal the signature of phase separation.

force for the onset of phase separation through spinodal decomposition. We suggest that the formation of the fluctuating, transient bi-continuous phase is responsible for the occurrence of strong non-ideality in the SBBM.

\subsection{Free-energy diagram of spinodal decomposition}

There is no thermodynamic barrier to the reaction inside the spinodal region. Thus, the process of spinodal decomposition can be treated purely as a diffusion problem, and many of the characteristics of the spinodal decomposition can be obtained by an approximate analytical solution to a generalized diffusion equation describing motion on an external potential.

As a special case of phase transition, spinodal decomposition can be shown on a phase diagram exhibiting a miscibility gap as shown in the figure 6 a. Thus, phase separation occurs whenever a system changes into the unstable region of the phase diagram (figure 6a). The boundary separating the metastable and the unstable region is called spinodal curve and can be obtained by performing a common tangent construction of the free-energy diagram. In the spinodal region, the curvature of the free-energy curve is negative.

If a binary mixture, kept at a high temperature $T_{1}$ is quenched (rapidly cooled) to a lower temperature $\mathrm{T}_{2}$, the composition that is initially same everywhere, starts to become inhomogeneous, as small fluctuation in composition that produces A-rich and B-rich regions will cause the total free energy to decrease. Therefore, 'uphill' diffusion takes place until the equilibrium compositions $\mathrm{X}_{1}$ and $\mathrm{X}_{2}$ are reached.

The free energy curve is plotted as a function of composition for the phase separation temperature $T_{2}$ as shown figure $6 \mathrm{~b}$. Equilibrium phase compositions are those corresponding to the free energy minima. Regions of negative curvature $\left(\mathrm{d}^{2} \mathrm{G} / \mathrm{dx}^{2}<0\right)$ lie within the inflection points of the curve $\left(d^{2} \mathrm{G} / \mathrm{dx}^{2}=0\right)$ which are called the spinodes (marked as S1 and S2 in figure 6a). To observe spinodal decomposition, a very fast transition, often called a quench, is required to move from the stable to the spinodally unstable region of the phase diagram.

\section{$3.7 \quad$ Non-ideality in terms of real binary mixture models at different mole fractions}

While theoretical development in the area of real world binary mixture is comparatively slow due to the enormous complexity associated with the intermolecular potential, our simple SBBM can explain several important structural aspects of them. The composite chemistry of binary solutions especially in terms of their nonideal behaviour observed in several experimental and simulation studies can also be correlated. Similar to the morphologies of our SBBM model, the real world aqueous amphiphilic solutions are also found to exhibit a wide range of structural variation depending on their binary composition. In recent years Raman and infrared study shed light on the effects of dimethyl sulphoxide (DMSO) on water structure leading to the conclusion that small amounts of dimethyl sulphoxide act as "structure breaker" in water. ${ }^{46}$ Accompanied with numerous experimental efforts, we have also investigated several static and dynamical properties of aqueous DMSO mixture and their concentration dependence, in our early simulation studies. ${ }^{47,48}$ All these studies result an anomalous composition range of water-DMSO binary mixture in the low DMSO concentration interval, especially when the mole fraction of DMSO $\left(\mathrm{x}_{\mathrm{DMSO}}\right)$ is in the range of $0.10-0.20$. The non-ideality has been reflected 


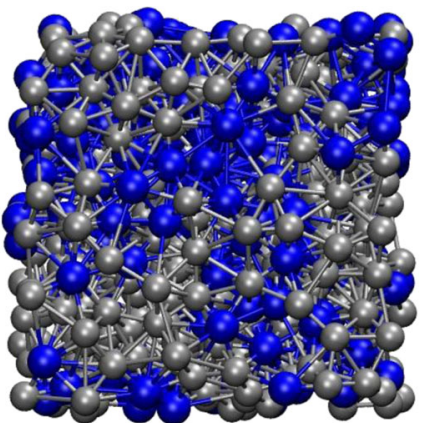

(a)

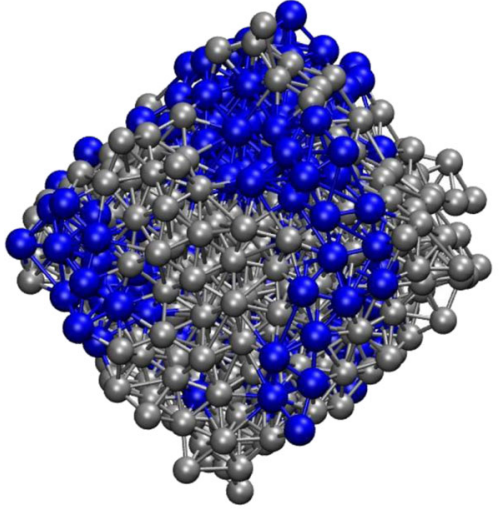

(b)

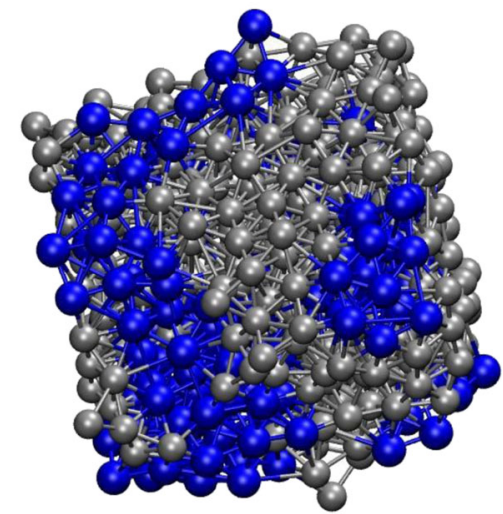

(c)

Figure 5. (a) Snapshot for structure breaker parent liquid with $n=500$ at solute composition $\mathrm{x}_{\mathrm{B}}=0.4$ and temperature $\mathrm{T}^{*}=1.6$. (b) and (c) Snapshots for the corresponding inherent structure in CPK mode of drawing method in graphical representation where silver (light gray) colour signifies solvent (A) and blue (dark gray) for solute (B). Note that the snapshots are taken from different angles. While figure 5a shows the parent structure to be almost homogeneous, figures $5 \mathrm{~b}$ and c clearly indicate a signature of spinodal decomposition.

in a number of physical properties such as, in diffusion coefficient of DMSO, in the average local composition fluctuation of water, in the mean square deviation of total dipole moment, in the orientational correlation function of $\mathrm{O}-\mathrm{H}$ bond of water, to mention a few.

In order to further establish the origin of non-ideality, we have evaluated the mean square fluctuation of the total energy of the system of water-DMSO mixture at their different compositions. The corresponding plot at various concentration of DMSO is shown in figure 7. We have obtained two different branches of energy fluctuation with a break at $10-15 \%$ mole percentage of DMSO. At that particular concentration interval, energy fluctuation becomes considerably restrained. This lowering of fluctuation implies a significant structural ordering emerging in the system. Around $20 \%$ of DMSO concentration, this fluctuation again increases following the ideal trend. Earlier, we have also observed similar behaviours in many other physical properties; for example, diffusion and composition fluctuation. ${ }^{47,48}$
Such a drop in energy fluctuation indeed arises due to the intervening structural phase transition by the formation of a spanning cluster of methyl groups of DMSO molecules assisted by surrounding water molecules.

Theoretical analyses of large scale computer simulation studies lead to the following molecular picture of the anomalous concentration range. In figure 8 we show the snapshots of observed configurations of water-DMSO mixture at two different compositions $\left(\mathrm{x}_{\mathrm{DMSO}}=0.1\right.$ and 0.15$)$. At extremely low concentration (from 0.05-0.10) methyl groups of DMSO form micelle-like islands those are segregated throughout the system (figure 8a). At relatively higher concentration (beyond 0.10) these DMSO islands start to form an extended aggregate associated with water, aided by both hydrophobic and H-bond interactions. Beyond a critical concentration (in case of water-DMSO, it is 0.15) they form a spanning cluster (figure $8 \mathrm{~b}$ ), via percolation transition. At this concentration range it clearly appears to be a phase separated bi-continuous binary solution 


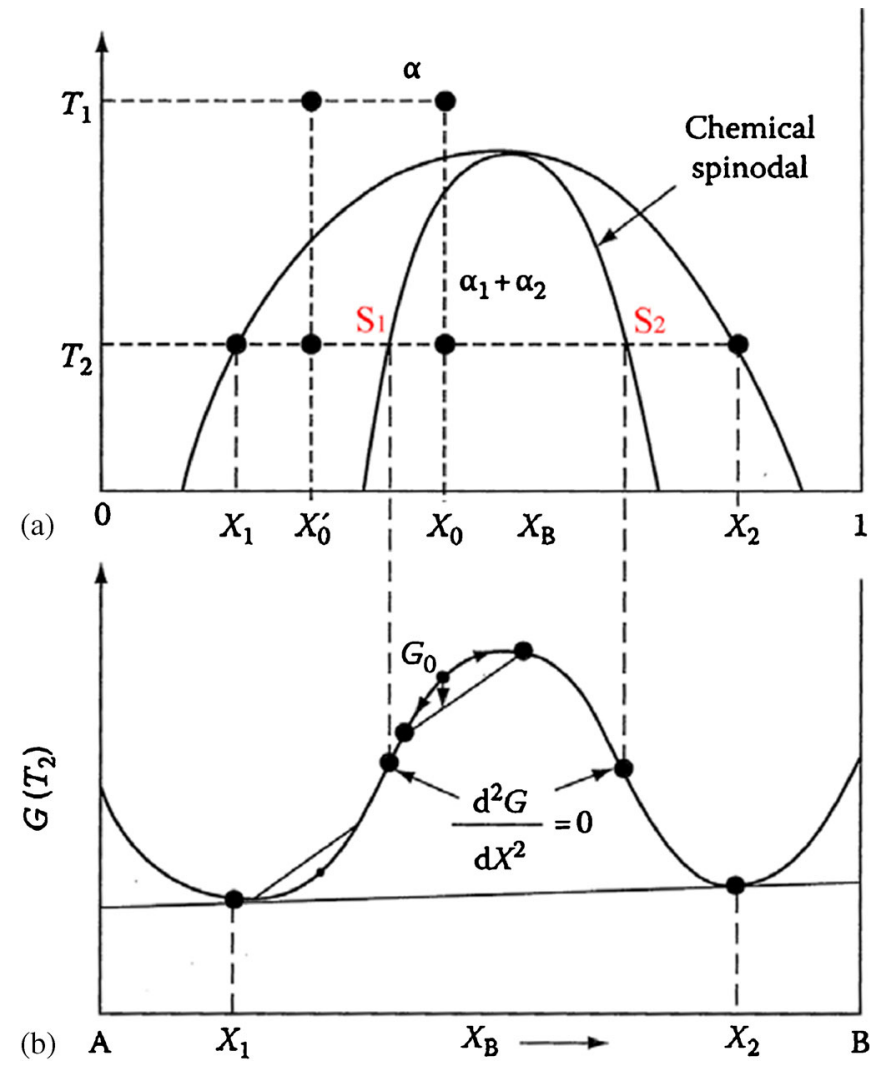

Figure 6. Schematic free energy diagram of the spinodal decomposition. (a) Spinodal decomposition is shown on the phase diagram displaying a miscibility gap. Note that the boundary separating the metastable and the unstable region is known as spinodal curve that can be obtained by performing a common tangent construction of the free-energy curve. (b) The free energy curve is plotted as a function of composition for the phase separation temperature $T_{2}$. In the spinodal region, the curvature of the free-energy curve is negative. Note that the binary mixture with initial temperature $\left(T_{1}\right)$ is cooled quickly to a lower temperature $\left(T_{2}\right) . X_{1}$ and $\mathrm{X}_{2}$ are the equilibrium compositions that correspond to the free energy minima. The spinodes are marked as S1 and S2 in the diagram. In the spinodal region, the curvature of the free-energy plot is negative.

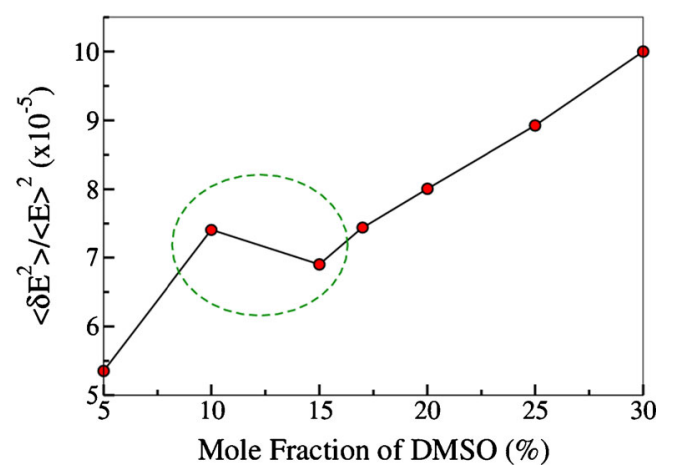

Figure 7. Effect of DMSO concentration on mean square fluctuation of total energy (E) of the system. A nonmonotonicity in the concentration dependence of energy fluctuation is observed at $10 \%-15 \%$ mole percentage of DMSO. which is rather similar to our SBBM model. Such aggregation phenomenon seems to be quite general for such aqueous amphiphilic solutions where competetitive hydrophobic and hydrophilic interactions determine the structural and dynamical ordering of the whole system.

Similar phenomenon is observed for another amphiphilic solute ethanol. ${ }^{49-51}$ Several rigorous experimental studies ${ }^{52,53}$ have pointed out a host striking anomalies in a number of thermodynamic and transport properties at low concentration of ethanol. In order to understand the origin of the anomalous behaviour of water-ethanol binary mixture we probed the local structure of the system. ${ }^{54}$ The existence of these composition dependent anomalies were found in many thermophysical properties, such as local composition fluctuation, radial distribution function of ethyl groups, diffusion co-efficient, especially at small length scales. The microheterogenity present in the system at low ethanol concentration was further quantified by using different methods for detecting percolation. We find that there are small clusters or microaggregates of ethanol molecules at low concentrations, and the size of the clusters gradually increase as the concentration of ethanol is increased. This transition from microaggregates to a bi-continuous phase shows strong signatures of percolation of the ethanol clusters - the network being formed by the ethyl groups. It is very difficult to capture exact threshold in this type of complex binary liquids. However in case of aqueous ethanol mixture, there is a strong signature of percolation induced structural transition in the region $\mathrm{x}_{\mathrm{EtOH}} \approx 0.075-0.10$. The following snapshots (figure $8 \mathrm{c}$ and $8 \mathrm{~d}$ ) represent appearance of such percolation transition or spanning cluster formation through hydrophobic interaction of ethyl groups.

Related to the structural aspects observed in SBBM model, another amphiphilc molecule that exhibits structural morphogenesis is tertiary butyl alcohol (TBA) possessing the largest aliphatic group among monohydric alcohols that are miscible with water at any proportions. The hydrophobic interaction is, therefore, much higher than the other small molecules. This is manifested as a strong non-monotonic behaviour in many physical and thermodynamic behaviour, as well as spectral signatures of water-TBA binary mixtures. ${ }^{55-57}$ For example, partial molar volume shows sharp minimum at $\mathrm{x}_{\mathrm{TBA}}=0.03$, while heat capacity shows sharp maximum at $\mathrm{x}_{\mathrm{TBA}}=0.05$. In our earlier simulation studies, we have observed strong anomalies in local composition fluctuation, self-diffusion coefficient of TBA, peak height of radial distribution function of TBA molecules - all deviations manifested in the composition range 

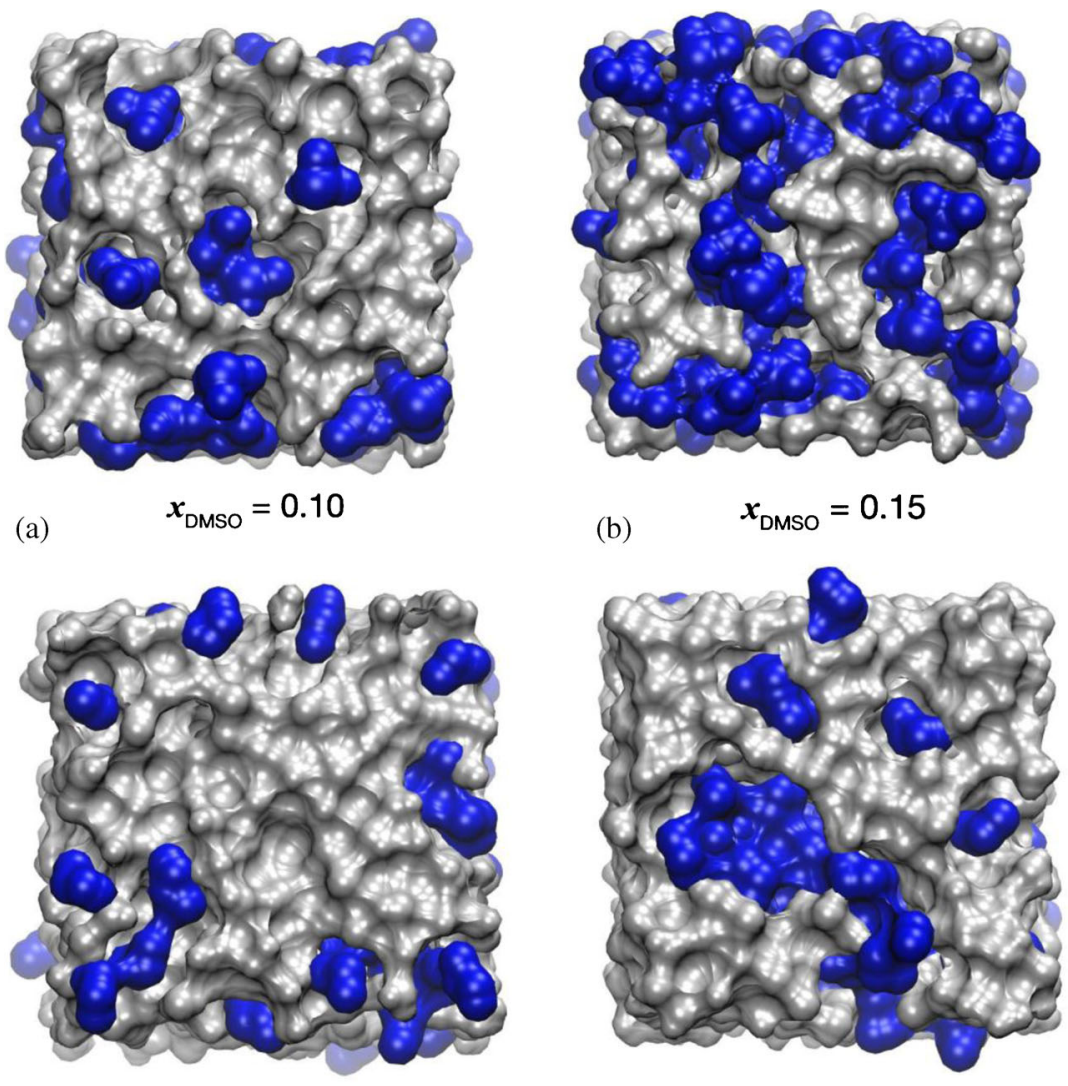

(c)

$$
x_{\text {eth }}=0.07
$$

(d)

$$
x_{\text {eth }}=0.10
$$
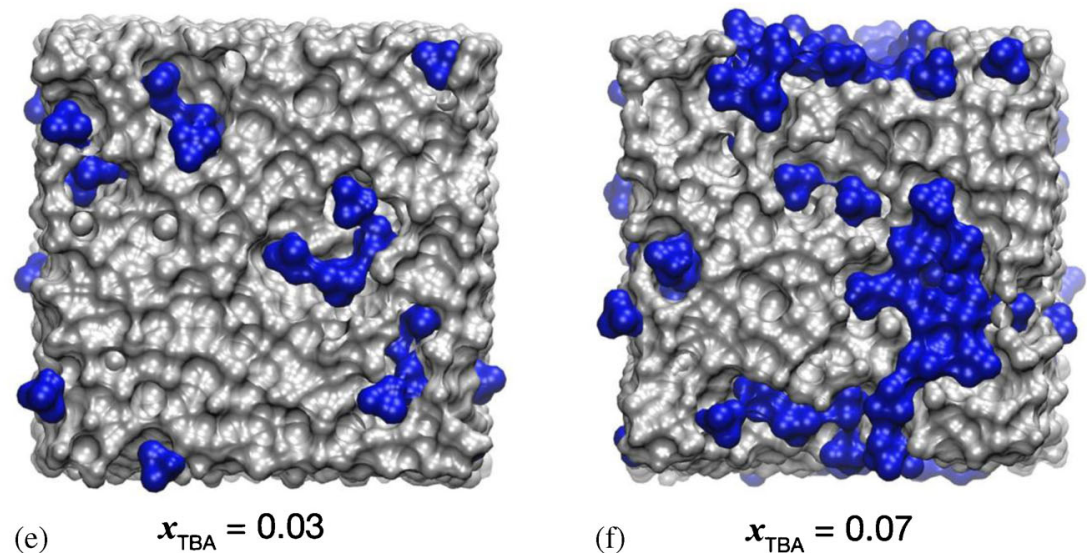

(e)

$$
x_{\text {TBA }}=0.03
$$

$$
\boldsymbol{x}_{\mathrm{TBA}}=0.07
$$

Figure 8. Snapshots of the simulation of different binary mixtures - waterDMSO as shown in (a), (b); water-ethanol as shown in (c), (d) and water-TBA as shown in (e), (f). Water molecules are shown in silver colour. Co-solvents (DMSO, ethanol and TBA) are represented in blue colour. Note that we present the snapshots at two different concentrations for each case - one before the onset of percolation to show microheterogeneity in the system, and one after the onset of percolation to show the spanning cluster of the co-solvent.

$\mathrm{x}_{\mathrm{TBA}}=0.05-0.07 .^{55}$ These anomalies were found to be related to the formation of a dynamic and fluctuating bi-continuous phase of TBA clusters and water clusters. The TBA molecules form segregated clusters at low concentrations below $\mathrm{x}_{\mathrm{TBA}}=0.05$. Above the critical concentration, the TBA molecules start forming spanning clusters, indicating a percolation transition, as shown in figure $8 \mathrm{e}$ and $8 \mathrm{f}$. The SBBM model closely resembles this structural transformation. The percolation transition observed in TBA is sharp and the threshold is quantified to be at $\mathrm{x}_{\mathrm{TBA}}=0.05$. The striking similarity of water-TBA binary mixture to water-DMSO 
and water-ethanol solutions points to the possibility of a unified understanding of the anomalies in all these binary mixtures, in terms of structural transformations. The formation of transient, fluctuating bi-continuous phase in the SBBM model not only gives us confidence about such unified understanding, but also opens up a new avenue towards interpretation of the non-ideality in terms of the dynamics of microscopic phase separation.

\section{Conclusions}

Let us summarize the main results of this paper. We have first discussed how non-ideality in a structure breaking model liquid can be attributed to a phase separation in its inherent structure. Due to fluctuations, the transient phase separation configurations are unstable at normal temperatures, thereby making their detection practically impossible. Nevertheless, well-defined phase separation in the inherent structure is sufficient reason to interpret non-ideality in terms of the dynamics of phase separation. Note that for structure forming binary liquids (where the two constituents like each other) we find no such phase separation in the inherent structures. The non-ideality in the structure forming liquid is of different kind, and be easily understood from reinforcement of structure. It does not have the possibility of phase separation. Note the difference between a microscopic interpretation, such as lowering of pair correlation function and a macroscopic explanation that is invoked here for structure breaking liquid.

The situation in the real world of amphiphilic solute molecules in water is far more complex than our simple SBBM model system. Here we can imagine that water molecules play the role of A but the assignment of the amphiphilic solute is more complex. In the case of DMSO, the strength of the hydrogen bond between the oxygen atom of $>\mathrm{S}=\mathrm{O}$ with the hydrogen atom of water molecules is stronger than the hydrogen bond between two water molecules. Therefore, the scenario is set for structure making mixture. But the methyl groups of DMSO (as also of TBA and the ethyl group of EtOH) dislike water molecules. Thus the situation is far more complex. However, we can draw important lessons as discussed above and reiterated below.

For the amphiphilic solutes like DMSO, ETOH, TBA, dioxane and many such small amphiphilic solutes, the phase separation dynamics is more intricate but a phase separation is certainly possible. The possibility increases because the hydrophobic groups can join together while the hydrophilic groups can form bonds with water molecules, or even among themselves as in $\mathrm{EtOH}$.
We indeed find strong signatures of the formation of transient bi-continuous phase when we study the quenched structures obtained by removing the vibrational motion of the molecules. This allows the study of static structures. We find that such a structure indeed support the formation of bi-continuous phase. We have shown snaps of such structures for DMSO, EtOH and TBA.

Thus, the bicontinuous phase formations in these aqueous mixtures are driven by complex interactions. But our main hypothesis that expresses the occurrence of strong non-ideality in these mixtures is due to transient phase separations. This can be further verified by calculation of the inherent structures of these systems.

An intriguing anomaly observed in many binary systems is a crossover from structure breaking to structure making scenario and vice versa, with increasing solute composition. This can be explained in terms of the formation and breakdown of the bicontinuous phase mentioned above.

\section{Supplementary Information}

Single particle potential energy distribution of parent liquid for structure former binary mixture (Model I) at mole fraction $\left(\mathrm{x}_{\mathrm{B}}\right)=0.4$ and temperature $\left(\mathrm{T}^{*}\right)=1$ is shown in figure $\mathrm{S} 1$ and the corresponding single particle inherent structure energy distribution is shown in figure S2. Supplementary information is available at www.ias.ac.in/chemsci.

\section{Acknowledgement}

This work was supported in parts by grants from the Department of Science \& Technology (DST). BB thanks DST for a JC Bose Fellowship.

\section{References}

1. Bagchi B 2012 In Molecular Relaxation in Liquids (USA: Oxford University Press)

2. Bagchi B 2013 In Water in Biological and Chemical Processes: From Structure and Dynamics to Function (London UK: Cambridge University Press)

3. Luzar A and Chandler D 1993 J. Chem. Phys. 988160

4. Chattoraj S, Chowdhury R, Ghosh S and Bhattacharyya K 2013 J. Chem. Phys. 138214507

5. Chandra A and Bagchi B 1991 J. Chem. Phys. 948367

6. A Chandra and B. Bagchi 1989 J. Chem. Phys. 911829

7. Gupta R and Chandra A 2007 J. Chem. Phys. 127 024503

8. Chowdhuri S and Chandra A 2005 J. Chem. Phys. 123 234501 
9. Roshkovskii G V, Ovchinnikova R A and Penkina N V 1982 Zh. Prikl. Khim. 551858

10. Mazurkiewicz J and Tomasik P 1990 J. Phys. Org. Chem. 3493

11. Kaatze U, Brai M, Sholle F-D, Pottel R 1990 J. Mol. Liq. 44197

12. Mukherjee A, Srinivas G and Bagchi B 2001 Phys. Rev. Lett. 865926

13. Mukherjee A and Bagchi B 2001 J. Phys. Chem. B 105 9581

14. Calvo F, Bogdan T V, de Souza V K and Wales D J 2007 J. Chem. Phys. 127044508

15. Middleton T F, Rojas J H, Mortenson P N and Wales D J 2001 Phys. Rev. B 64184201

16. Heuer A 1997 Phys. Rev. Lett. 784051

17. Sastry S 2000 Phys. Rev. Lett. 85590

18. Chakrabarti D and Bagchi B 2006 PNAS 1037217

19. Sastry S 2001 Nature 409164

20. Sastry S, Debenedetti P G and Stillinger F H 1997 Phys. Rev. E 565533

21. Sastry S, Debenedetti P G and Stillinger F H 1998 Nature 393554

22. Stillinger F H and Weber T A 1984 Science 225983

23. Stillinger F H 1995 Science 2671935

24. Weber T A and Stillinger F H 1985 Phys. Rev. B 311954

25. Zwanzig R 1995 PNAS 929801

26. Zwanzig R, Szabo A and Bagchi B 1992 PNAS 8920

27. Zwanzig R 1988 PNAS 852029

28. Bryngelson J D and Wolynes P G 1989 J. Phys. Chem. 936902

29. Binder K and Stauffer D 1974 Phys. Rev. Lett. 331006

30. Siggia E D 1979 Phys. Rev. A 20595

31. Koga T and Kawasaki K 1993 Physica A 196389

32. Gunton J D, San Miguel M and Sahni P S 1983 In Phase transitions and critical phenomena Domb $\mathrm{C}$ and Lebowitz J L (ed.) (London: Academic) 8

33. Santra M and Bagchi B 2009 J. Chem. Phys. 131084705

34. Mao Z, Sudbrack C K, Yoon K E, Martin G and Seidman D N 2007 Nature Mater. 6210

35. Talanquer V and Oxtoby D W 1998 J. Chem. Phys. 109 223

36. Peters J F and Berney E S IV 2010 J. Geotech. Geoenviron. Eng. 136310
37. Wang J, McLaughlin I L and Silbert M 1991 J. Phys.: Condens. Matter 35603

38. Mitra M K and Muthukumar M 2010 J. Chem. Phys. 132 184908

39. Cahn J W and Hilliard J E 1958 J. Chem. Phys. 28258

40. Langer J S, Bar-on M and Miller H D 1975 Phys. Rev. A 111417

41. Sarkar S and Bagchi B 2011 Phys. Rev. E 83031506

42. Abraham S E, Chakrabarty D and Bagchi B $2007 \mathrm{~J}$. Chem. Phys. 126074501

43. Press W H, Flannery B P, Teukolsky S A and Vetterling W T 1990 In Numerical Recipes in FORTRAN (Cambridge: Cambgidge University Press)

44. Sarkar S, Biswas R, Santra M and Bagchi B 2013 Physical Review E $\mathbf{8 8} 022104$

45. Humphrey W F, Dalke A and Schulten K 1996 J. Mol. Graphics 1433

46. Bertoluzza A, Bonora S, Battaglia M A and Monti P 2005 J. Raman Spec. 8231

47. Roy S, Banerjee S, Biyani N, Jana B and Bagchi B 2011 J. Phys. Chem. B 115685

48. Banerjee S, Roy S and Bagchi B 2010 J. Phys. Chem. B 11412875

49. Pradhan T Ghoshal P and Biswas R 2008 J Chem Sci. 120275

50. Mijaković M, Kežić B, Zoranić L, Sokolić F, Asenbaum A, Pruner C, Wilhelm E and Perera A 2011 J. Mol. Liq. 16466

51. Dixit S, Crain J, Poon W C K, Finney J L and Soper A K 2002 Nature 416829

52. Juurinen I, Nakahara K, Ando N, Nishiumi T, Seta H, Yoshida N, Morinaga T, Itou M, Ninomiya T, Sakurai Y, Salonen E, Nordlund K, Hämäläinen K and Hakala M 2011 Phys. Rev. Lett. 107197401

53. Wakisaka A and Matsuura K 2006 J. Mol. Liq. 12925

54. Banerjee S, Ghosh R and Bagchi B 2012 J. Phys. Chem. B 1163713

55. Banerjee S, Furtado J and Bagchi B 2014 J. Chem. Phys. 140194502

56. Pradhan T Ghoshal P and Biswas R 2008 J. Phys. Chem. A 112915

57. Gazi H A R and Biswas R 2011 J. Phys. Chem. A 115 2447 